\title{
BMJ Open Effect of resting heart rate on the risk of all-cause death in Chinese patients with hypertension: analysis of the Kailuan follow-up study
}

\author{
Mao Xiang Zhao (D) , ${ }^{1}$ Quanhui Zhao, ${ }^{2}$ Mengyi Zheng, ${ }^{3}$ Tong Liu, ${ }^{3}$ Yao Li, ${ }^{1}$ \\ Miao Wang, ${ }^{1}$ Siyu Yao, ${ }^{1}$ Chi Wang, ${ }^{1}$ Yan-Ming Chen, ${ }^{1}$ Hao Xue, ${ }^{1}$ Shouling $W u^{2}$
}

To cite: Zhao MX, Zhao $Q$, Zheng M, et al. Effect of resting heart rate on the risk of all-cause death in Chinese patients with hypertension: analysis of the Kailuan follow-up study. BMJ Open 2020;10:e032699. doi:10.1136/ bmjopen-2019-032699

- Prepublication history for this paper is available online. To view these files, please visit the journal online (http://dx.doi org/10.1136/bmjopen-2019032699).

Received 04 July 2019 Revised 11 December 2019 Accepted 08 January 2020

Check for updates

(c) Author(s) (or their employer(s)) 2020. Re-use permitted under CC BY-NC. No commercial re-use. See rights and permissions. Published by BMJ.

${ }^{1}$ Department of Cardiology, Chinese PLA General Hospital, Beijing, China

${ }^{2}$ Department of Cardiology, Kailuan General Hospital, Tangshan, Hebei, China ${ }^{3}$ North China University of Science and Technology, Tangshan, Hebei, China

Correspondence to

Dr Hao Xue;

xuehaoxh301@163.com

\section{ABSTRACT}

Objective Previous studies have shown that an elevated heart rate is associated with a higher risk of cardiovascular events. This study aimed to prospectively examine the relationship between resting heart rate (RHR) and allcause mortality in Chinese patients with hypertension. Design An observational, prospective and populationbased cohort study.

Setting The Kailuan cohort study was conducted in Tangshan City in northern China.

Participants We enrolled 46561 patients who did not receive beta-blocker treatment and were diagnosed with hypertension for the first time during an employee health examination in Kailuan Group in 2006 and 2008.

Outcome The primary outcome of this study was allcause mortality.

Methods The patients in this study were followed for $9.25 \pm 1.63$ years. All patients were followed up face to face every 2 years. According to the distribution of RHR in the study population, RHR was categorised into five groups on the basis of quintiles: Q1: RHR $\leq 68$ beats per minute (bpm); Q2: RHR >68 and $\leq 72$ bpm; Q3: RHR > 72 and $\leq 76$ bpm; Q4: RHR > 76 and $\leq 82$ bpm; Q5: RHR > 82 bpm. Cox proportional hazards model, which was adjusted for traditional risk factors, was used

Results During follow-up, 4751 deaths occurred. After adjustment for potential confounders, restricted cubic spline regression showed that the risk of all-cause mortality increased with heart rate. In multivariate Cox regression analyses adjusted for age, sex and major covariates, the HR for all-cause mortality was $1.31(95 \% \mathrm{Cl}$ 1.27 to 1.33 ) in the highest quintile group (Q5) compared with the lowest quintile group (Q1).

Conclusion An increase in RHR is a long-term risk factor of all-cause mortality in Chinese patients with hypertension.

Trial registration number ChiCTR-TNC-11001489.

\section{INTRODUCTION}

Cardiovascular disease (CVD) is the leading cause of death in China. ${ }^{1}$ At least half of cardiovascular deaths are associated with hypertension each year. ${ }^{2}$ Although more attention has been paid to control blood pressure in recent years, CVD mortality continues
Strengths and limitations of this study

- Our study was based on the Kailuan study, which was a prospective, population-based cohort study with a large sample size.

- This is the first prospective study to examine the relationship between resting heart rate (RHR) and the risk of all-cause mortality in Chinese patients with hypertension.

- Given that beta-blockers have an effect on RHR, we exclude patients who received beta-blocker treatment.

- Death due to cardiovascular disease was not documented.

- We did not include all changes that occurred during follow-up, and baseline RHR might have changed over the long follow-up duration.

to increase. ${ }^{2}$ This discrepancy implies that, in addition to blood pressure, other factors may be involved in the increasing CVD mortality.

Sympathetic overactivity is involved in the pathogenesis of hypertension. An increase in blood pressure is also closely related to an increase in heart rate. ${ }^{3}$ Resting heart rate (RHR) is a non-invasive physiological indicator which reflects the activity of the autonomic nervous system. Heart rate is an easier and more direct way to determine health status compared with body mass index $(\mathrm{BMI})$, smoking and waist circumference. ${ }^{4-6}$ Several studies have suggested that RHR is an independent risk factor of all-cause mortality in patients with hypertension, and patients with an RHR $>80$ beats per minute (bpm) have a significantly increased risk of all-cause mortality. ${ }^{7-17}$ The 2018 European Society of Cardiology/European Society of Hypertension $(\mathrm{ESC} / \mathrm{ESH})$ guidelines for management of arterial hypertension proposed an RHR $>80 \mathrm{bpm}$ as a factor affecting cardiovascular risk in patients with hypertension. ${ }^{17}$ The 
Chinese Expert Consensus on Heart Rate Management for Hypertensive Patients also suggested to set $80 \mathrm{bpm}$ as the cut-off point for heart rate intervention in patients with hypertension. ${ }^{18}$ An association between RHR and all-cause mortality has been reported in Europe ${ }^{17}$ and the USA. ${ }^{11}$ However, the effect of elevated RHR on the risk of all-cause mortality has not been studied in Chinese patients with hypertension. Therefore, to address this issue, we prospectively investigated the relationship between RHR and all-cause mortality in the Kailuan cohort study.

\section{METHODS}

\section{Patient and public involvement}

Patients and the public were not involved in the design of this study.

\section{Study design and participants}

Data were derived from the Kailuan cohort study, which was a large, observational, prospective and populationbased cohort study that was carried out from June 2006 to October 2007. A total of 101510 men and women (referred to as the 'original cohort') were enrolled in Tangshan City in northern China. ${ }^{19} 20$ The design, methods, rationale and examination details of the Kailuan cohort study were previously published elsewhere. ${ }^{19} 21$ Participants were then followed biennially with repeated questionnaires and medical examinations via face-to-face interviews with medical staff and trained research nurses..$^{2122}$ In the current analysis, participants were eligible if they took part in a medical examination for the first time in either the 2006-2007 or the 2008-2009 examination and had hypertension (blood pressure $\geq 140 / 90 \mathrm{~mm} \mathrm{Hg}$, currently on antihypertensive therapy or a physician's diagnosis). Patients with arrhythmia (including atrial fibrillation, atrial flutter, atrial premature beat, ventricular ectopic beats and atrioventricular block) or those taking betaadrenergic blocking agents were excluded.

\section{Data collection and assessment of potential confounding covariates}

As described in detail previously, ${ }^{19} 212324$ BMI was calculated as weight divided by height $\left(\mathrm{kg} / \mathrm{m}^{2}\right)$. Data on baseline variables, including age, sex, smoking habits, drinking status and physical activity, were ascertained from a standard questionnaire. Diabetes mellitus was defined as a fasting blood glucose level $\geq 7.0 \mathrm{mmol} / \mathrm{L}$, taking oral hypoglycaemic agents or insulin, or a self-reported physician diagnosis. A history of stroke and myocardial infarction was determined by a self-reported physician diagnosis. Biochemical parameters, including fasting blood glucose, triglyceride, total cholesterol and high-density lipoprotein cholesterol levels, were measured using an autoanalyser (Hitachi 747; Hitachi, Tokyo, Japan) at the central laboratory of Kailuan General Hospital.

\section{RHR measurements}

RHR was measured via a 12-lead ECG (ECG9130P; Nihon Kohden, Tokyo, Japan) at baseline with participants resting in supine position for at least $5 \mathrm{~min}$. The inverse of the interval between R-waves for five consecutive QRS complexes was used to determine heart rate.

\section{Blood pressure measurements}

Blood pressure was measured twice, at a 5 min interval, on the left arm with participants in a seated position after at least 5 min of rest, using a mercury sphygmomanometer. Hypertension was defined as self-reported use of antihypertensive medication, history of hypertension, systolic blood pressure $\geq 140 \mathrm{~mm} \mathrm{Hg}$ or diastolic blood pressure $\geq 90 \mathrm{~mm} \mathrm{Hg}$.

\section{Outcome}

The primary outcome of this study was all-cause mortality. The determination of death was described in detail previously. ${ }^{19}$ Participants were followed from the end point of the first examination until death or end of follow-up (31 December 2016), whichever event occurred first. Participants underwent clinical examination biennially, and information on any fatal events was collected through review of death certificates from provincial vital statistics offices, hospital records, medical insurance data, and interviews with next of kin, relatives or eyewitnesses, where such were possible. ${ }^{19}$ Vital status was determined by a review committee by 31 December 2016 .

\section{Statistical analysis}

Medical data were obtained from each participating hospital and stored in the study database (Oracle 10.2g) that is hosted in the server at Kailuan General Hospital. According to the distribution of RHR in the study population, participants were categorised into five groups on the basis of quintiles: Q1: RHR $\leq 68 \mathrm{bpm}$; Q2: RHR $\geq 69$ and $\leq 72 \mathrm{bpm}$; Q3: RHR $>72$ and $\leq 76 \mathrm{bpm}$; Q4: RHR $>76$ and $\leq 82 \mathrm{bpm}$; Q5: RHR $>82 \mathrm{bpm}$. All statistical analyses were conducted using SAS V.9.3. Continuous variables are shown as mean $\pm \mathrm{SD}$ and categorical variables are presented as percentages. Characteristics of the participants among the five RHR groups were compared using analysis of variance for continuous variables and the $\chi^{2}$ test for categorical variables. The cumulative incidence of end point events among the subgroups was estimated using the Kaplan-Meier method and compared using the log-rank test. Cox proportional hazard models were used to analyse the association between the five RHR subgroups and the risk of all-cause death, with adjustment for confounding variables. Antihypertensive drugs may have additional effects on all-cause death. Therefore, we performed sensitivity analyses by excluding participants using antihypertensive drugs during 2006-2008. For further analysis to investigate the relationship between RHR as a continuous variable and all-cause mortality, we used restricted cubic spline regression to calculate the HRs and their 95\% CIs. All statistical tests were two-sided 


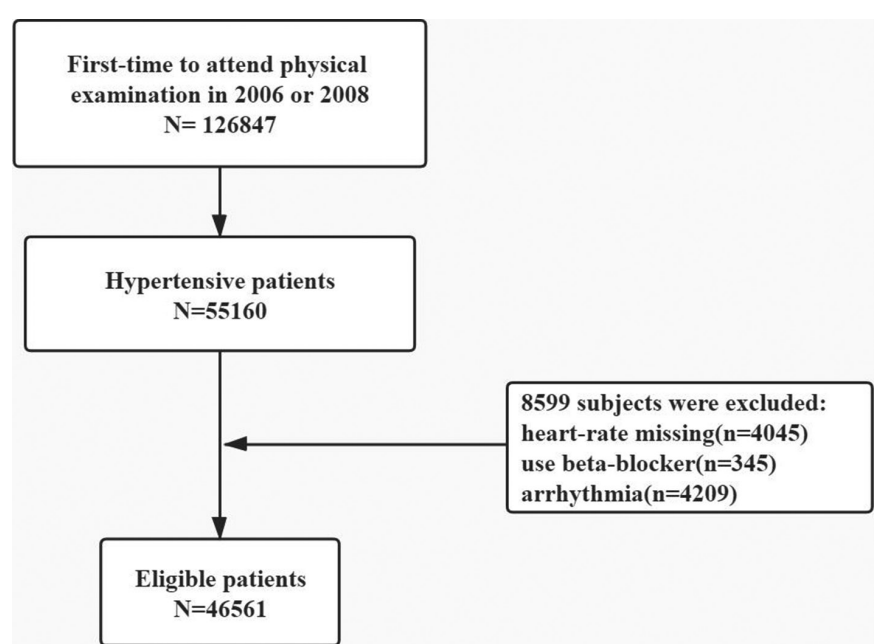

Figure 1 Flow chart of the Kailuan cohort study.

and differences were considered statistically significant at $\mathrm{p}<0.05$.

\section{RESULTS}

\section{Baseline characteristics of the study population}

Among 126847 individuals in the original cohort, 55160 were hypertensive. Among the remaining participants, 4554 who experienced arrhythmia or started taking betablockers and 4045 with missing information on RHR were excluded. A total of 46561 participants were therefore included in the current analysis (figure 1). The mean age of the participants was $54.42 \pm 11.41$ years and the average RHR was $75.19 \mathrm{bpm}$. Among the patients, 39963 (85.8\%) were men and $6598(14.2 \%)$ were women. Participants with a higher heart rate were more likely to have diabetes mellitus, higher fasting blood glucose levels, total cholesterol levels, systolic blood pressure and diastolic blood pressure $(\mathrm{p}<0.01)($ table 1$)$.

\section{Incidence of events}

The number of deaths and the incidence of mortality in each quintile of RHR are shown in table 2. In the mean follow-up period of $9.25 \pm 1.63$ years, a total of 4751 allcause deaths occurred. The number of all-cause deaths and cumulative incidence in Q1, Q2, Q3, Q4 and Q5 was $1048(10.13 \%), 638(8.41 \%), 875$ (9.63\%), $1062(10.49 \%)$ and $1128(11.99 \%)$, respectively (table 2 ). The log-rank test showed that there was a significant difference among the five subgroups $(\mathrm{p}<0.05)$ (figure 2$)$.

\section{RHR and risk of all-cause mortality}

In model 1, patients in the highest RHR quartile had a higher risk of mortality compared with those in the lowest quartile (unadjusted HR: 1.44, 95\% CI 1.32 to 1.56). After accounting for sociodemographic and cardiovascular risk factors (age, sex, low-density lipoprotein, high-density lipoprotein, triglycerides, physical activity, drinking, smoking, BMI, systolic blood pressure, history of stroke, myocardial infarction, diabetes mellitus and antihypertensive agent status), similar associations between the RHR categories and all-cause mortality were attenuated, but remained significant in model 3 (adjusted HR: 1.32, 95\% CI 1.21 to 1.45 ) (table 2).

The HR $(95 \% \mathrm{CI})$ for all-cause mortality was estimated by restricted cubic spline regression for RHR and the results are plotted in figure 3 . An RHR $\geq 76 \mathrm{bpm}$ was associated with a higher risk of all-cause death. The restricted

\begin{tabular}{|c|c|c|c|c|c|c|c|}
\hline & Q1 $(n=10349)$ & Q2 (n=7589) & Q3 (n=9086) & Q4 ( $n=10127)$ & Q5 (n=9410) & $F / \chi^{2}$ & $P$ value \\
\hline Age (years) & $55.95 \pm 11.18$ & $52.51 \pm 11.25$ & $54.29 \pm 10.97$ & $53.37 \pm 11.27$ & $53.40 \pm 11.72$ & 118.39 & $<0.01$ \\
\hline RHR (beats per minute) & $63.23 \pm 4.36$ & $70.07 \pm 0.41$ & $73.14 \pm 1.35$ & $78.66 \pm 1.66$ & $90.73 \pm 8.00$ & 53523.5 & $<0.01$ \\
\hline SBP $(\mathrm{mm} \mathrm{Hg})$ & $145.69 \pm 16.86$ & $145.88 \pm 16.90$ & $147.09 \pm 17.57$ & $148.12 \pm 17.58$ & $150.47 \pm 18.91$ & 109.05 & $<0.01$ \\
\hline $\mathrm{TG}(\mathrm{mmol} / \mathrm{L})$ & $1.74 \pm 1.34$ & $1.93 \pm 1.53$ & $1.84 \pm 1.45$ & $1.90 \pm 1.51$ & $2.04 \pm 1.69$ & 49.19 & $<0.01$ \\
\hline LDL-C (mmol/L) & $2.38 \pm 1.00$ & $2.51 \pm 0.94$ & $2.51 \pm 0.99$ & $2.58 \pm 0.99$ & $2.48 \pm 0.92$ & 53.45 & $<0.01$ \\
\hline $\mathrm{HDL}-\mathrm{C}(\mathrm{mmol} / \mathrm{L})$ & $1.56 \pm 0.44$ & $1.58 \pm 0.41$ & $1.54 \pm 0.43$ & $1.53 \pm 0.40$ & $1.58 \pm 0.44$ & 20.69 & $<0.01$ \\
\hline FBG (mmol/L) & $5.43 \pm 1.47$ & $5.55 \pm 1.61$ & $5.67 \pm 1.74$ & $5.80 \pm 1.86$ & $6.15 \pm 2.32$ & 207.52 & $<0.01$ \\
\hline Diabetes (\%) & 8.67 & 9.53 & 10.32 & 12.63 & 16.25 & 316.85 & $<0.01$ \\
\hline Antihypertensive medication (\%) & 24.60 & 18.05 & 22.23 & 21.77 & 21.39 & 98.01 & $<0.01$ \\
\hline History of myocardial infarction & 1.91 & 1.54 & 1.68 & 1.67 & 1.51 & 5.77 & 0.217 \\
\hline History of stroke & 4.10 & 2.95 & 3.21 & 3.23 & 3.00 & 25.55 & $<0.001$ \\
\hline
\end{tabular}

Q1: $R H R<68$ bpm; Q2: RHR >68 and $\leq 72$ bpm; Q3: RHR >72 and $\leq 76$ bpm; Q4: RHR >76 and <82 bpm; Q5: RHR >82 bpm.

BMI, body mass index; bpm, beats per minute; DBP, diastolic blood pressure; FBG, fasting blood glucose; HDL-C, high-density lipoprotein cholesterol; LDL-C, low-density lipoprotein cholesterol; RHR, resting heart rate; SBP, systolic blood pressure; TG, triglycerides. 
Table 2 HR (95\% Cl) for all-cause mortality according to resting heart rate quintiles

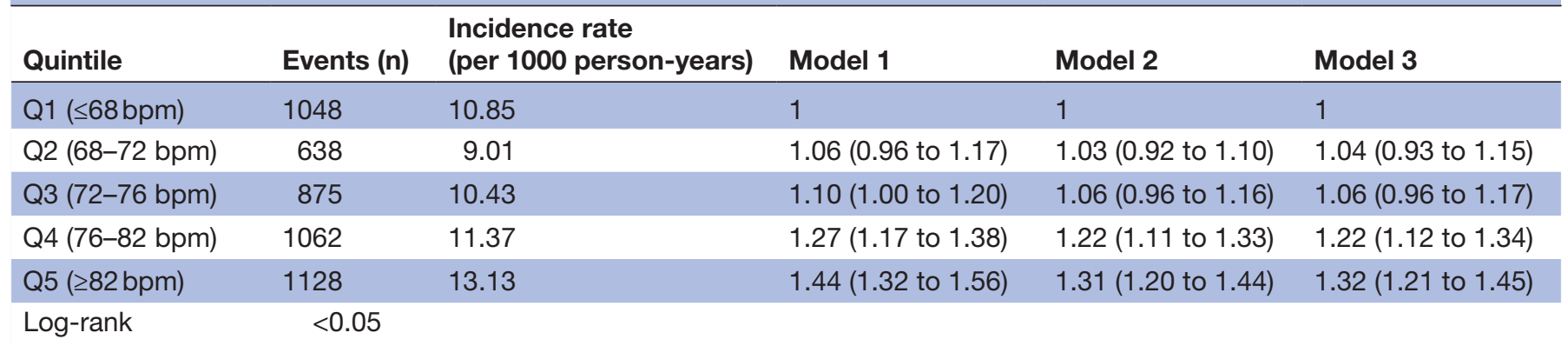

Model 1: adjusted for age and sex.

Model 2: adjusted for age, sex, low-density lipoprotein, high-density lipoprotein, triglycerides, physical activity, drinking, smoking and body mass index.

Model 3: adjusted for age, sex, low-density lipoprotein, high-density lipoprotein, triglycerides, physical activity, drinking, smoking, body mass index, systolic blood pressure, history of stroke, myocardial infarction, diabetes mellitus and antihypertensive agent status.

bpm, beats per minute.

cubic spline regression model showed a linear relationship between RHR and outcomes (figure 3).

\section{Sensitivity analysis}

We found that the results were robust after considering the effect of antihypertensive drugs. In the fully adjusted model, patients in the highest quintile of RHR had a $33 \%$ increased risk of mortality (HR: $1.33,95 \%$ CI 1.19 to 1.48 ) compared with those in the lowest quintile (table 3 ).

\section{DISCUSSION}

This study is the first prospective study to investigate the effect of RHR on all-cause mortality in a large-scale, Chinese population with hypertension. We found that the risk of all-cause mortality increased with an increase in RHR. Therefore, an increase in RHR is a long-term

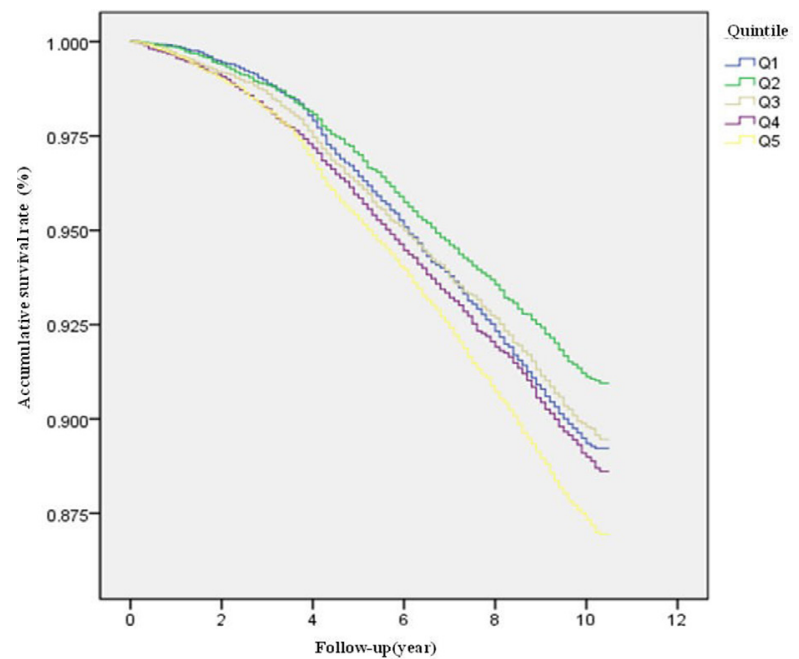

Figure 2 Kaplan-Meier survival curve for all-cause mortality stratified by $R H R$ levels. RHR quintiles are as follows: Q1: $\mathrm{RHR}<69$ bpm; Q2: RHR $\geq 69$ and <72 bpm; Q3: RHR $\geq 72$ and $<76$ bpm; Q4: RHR $\geq 76$ and <82 bpm; Q5: RHR $\geq 82$ bpm. bpm, beats per minute; $\mathrm{RHR}$, resting heart rate. risk factor of all-cause mortality in Chinese patients with hypertension.

RHR is an easily accessible clinical parameter. An elevated RHR is significantly associated with CVD and allcause mortality in healthy populations, ${ }^{25}$ in patients with chronic heart failure ${ }^{26}$ and in patients with atrial fibrillation. ${ }^{27}$ In our study, we found that elevated RHR was associated with an increased risk of all-cause mortality, and a linear-shaped relationship was found between RHR and all-cause mortality. The Framingham study, ${ }^{11}$ Glasgow study ${ }^{15}$ and French study ${ }^{12}$ also showed similar findings, where a relationship between increased RHR and allcause mortality was found. Moreover, we found that an RHR $\geq 76 \mathrm{bpm}$ and an RHR $\geq 82 \mathrm{bpm}$ were associated with a $21 \%$ and $31 \%$ increase in the risk of all-cause mortality compared with an RHR $<69$ bpm in Chinese patients with hypertension, respectively. Furthermore, restricted cubic

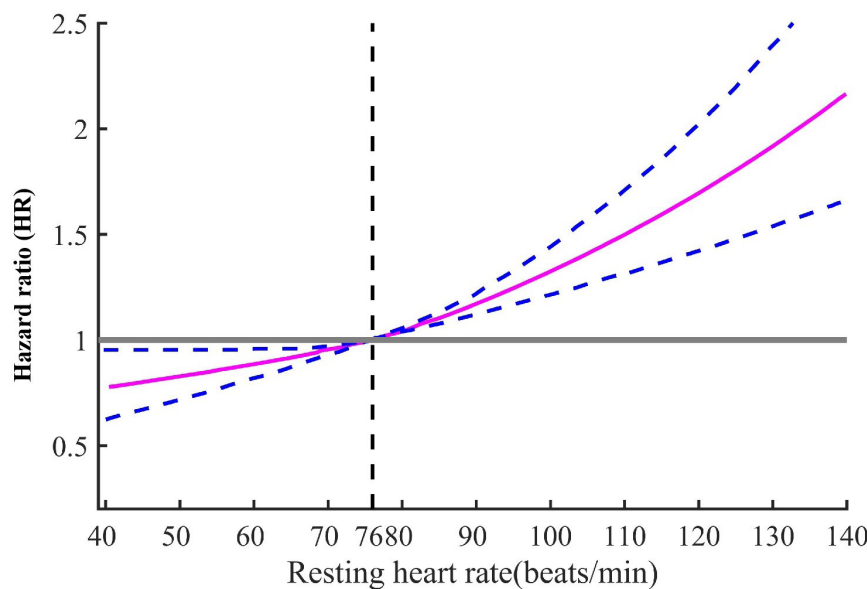

Figure 3 Cubic spline graph of adjusted $\mathrm{HR}$ and $95 \% \mathrm{Cl}$ for the association between RHR and all-cause mortality. The adjusted cubic spline model shows the relationship between RHR and all-cause mortality when an RHR of 76 beats per minute is the reference. The pink line shows the HR and the blue lines show the upper and lower $95 \%$ confidence limits. $\mathrm{RHR}$, resting heart rate. 
Table 3 HR (95\% Cl) of all-cause mortality excluding patients on antihypertensive treatment

\begin{tabular}{llll}
\hline & HR & $95 \%$ Cl & P value \\
\hline Q1 (RHR $\leq 68$ bpm) & 1.00 & & \\
Q2 (RHR $>68$ and $\leq 72$ bpm) & 1.01 & 0.88 to 1.14 & 0.97 \\
Q3 (RHR $>72$ and $\leq 76$ bpm) & 1.07 & 0.96 to 1.21 & 0.23 \\
Q4 (RHR $>76$ and $\leq 82$ bpm) & 1.21 & 1.08 to 1.35 & $<0.01$ \\
Q5 (RHR $>82$ bpm) & 1.33 & 1.19 to 1.48 & $<0.01$ \\
\hline
\end{tabular}

The model was adjusted for age, sex, low-density lipoprotein, high-density lipoprotein, triglycerides, physical activity, drinking, smoking, body mass index, systolic blood pressure, history of stroke, myocardial infarction, diabetes mellitus and antihypertensive agent status.

bpm, beats per minute; RHR, resting heart rate.

spline regression showed a linear relationship between RHR and risk of all-cause mortality, and an RHR $\geq 76 \mathrm{bpm}$ was associated with a higher risk for all-cause death. In fact, management of heart rate in patients with hypertension is also supported by the $2018 \mathrm{ESC} / \mathrm{ESH}$ guidelines for management of arterial hypertension. ${ }^{17}$ These guidelines suggest that an RHR $>80 \mathrm{bpm}$ affects cardiovascular risk in patients with hypertension. However, the latest Guidelines for the Prevention and Treatment of Hypertension in China $2018^{28}$ did not recommend elevated RHR as a prognostic cardiovascular risk factor in patients with hypertension, and the threshold value of RHR intervention was not mentioned. Therefore, further studies are required to examine the threshold value of RHR for heart rate intervention in Chinese patients with hypertension to reduce all-cause mortality.

In addition to Europe and Japan, ${ }^{29}$ national guidelines ${ }^{30-32}$ from other countries (including USA, Canada and UK) did not include heart rate as a risk factor for prognosis of CVD. Beta-blockers are the preferred drug to control heart rate in patients with high blood pressure. However, whether beta-blockers can be used as first-line antihypertensive drugs remains controversial in the guidelines from different countries. Hypertension management guidelines from China and Europe recommend beta-blockers as first-line medications, while the hypertension guidelines from the USA, ${ }^{31} \mathrm{UK}^{31}$ and Japan ${ }^{32}$ do not. One reason for the diversity in different guidelines is that beta-blockers (mainly atenolol) are significantly less effective than other types of blood pressure drugs (diuretics, ACE inhibitors, angiotensin receptor blockers, calcium channel blockers) in preventing stroke and cardiovascular events. ${ }^{33}$ However, unlike smoking, drinking, blood lipids and BMI, no results of heart rate intervention from randomised, clinical trials are available, which causes difficulty in providing treatment recommendations for patients with an elevated heart rate. Therefore, more randomised trials on heart rate intervention in patients with hypertension with a high heart rate are still required.

The mechanisms of RHR involved in the risk of mortality may be associated with sympathetic overactivity.
RHR is a non-invasive marker of autonomic nervous system function. Elevated RHR reflects a heightened sympathetic tone that contributes to vasoconstriction and insulin resistance by stimulating the alpha-adrenergic or beta-adrenergic receptors. These effects lead to increased blood pressure, lipid levels and blood sugar levels. ${ }^{35-39}$ However, an unbalanced autonomic nervous system is also related to inflammation and endothelial dysfunction. ${ }^{40}$ Therefore, elevated RHR could increase the risk of atherosclerosis, ${ }^{41}$ hyperinsulinaemia, ${ }^{42}$ myocardial ischaemia, hypertension ${ }^{43}$ and death in the long term. Additionally, Bangalore $e t a l^{44}$ provided strong evidence that beta-blocker intervention reduced sympathetic nerve activity and slowed heart rate. This condition is conducive to improving prognosis of CVD in myocardial infarction, sudden death and heart failure, and reducing mortality.

This study has several strengths, including its large sample size with a long follow-up of $9.25 \pm 1.63$ years. Additionally, this is the first prospective study to examine the relationship between RHR and risk of all-cause mortality in Chinese patients with hypertension. We also acknowledge several limitations in our study. First, our study had an unbalanced distribution of sex and most of the participants were male coal miners. However, sex distribution in our study was representative of the whole population of Kailuan Group. Second, our study was a single-centre, observational study. However, we prospectively observed participants for longer than 9 years of follow-up, and the data were analysed by adjusting for potential risk factors of CVD. Third, death due to CVD was not documented.

\section{CONCLUSION}

An increase in RHR may be an independent risk factor of all-cause mortality in Chinese patients with hypertension. Measurement of heart rate should be included in the overall assessment of patients with hypertension. This study provides a basis for management of heart rate in Chinese patients with hypertension. The cut-off point for heart rate interventions to reduce risk of allcause mortality in patients with hypertension needs to be further investigated in prospective studies.

Acknowledgements We thank all members of the Kailuan Study Group for their contribution and the participants who contributed their data.

Contributors MXZ, QZ, MZ, TL, Y-MC, SY, CW, YL and MW contributed to the concept of the study. SW and HX supervised the study. MXZ, QZ, MZ and TL conducted the data analysis.

Funding This work was supported by the National Nature Science Foundation of China (81570383).

Competing interests None declared.

Patient consent for publication Not required.

Ethics approval The Kailuan cohort study protocol was approved by the Medical Ethics Committee.

Provenance and peer review Not commissioned; externally peer reviewed.

Data availability statement Data may be obtained from a third party and are not publicly available. Our data were based on the Kailuan cohort study and are not publicly available. Please contact SW (email: drwusl@163.com) for details. 
Open access This is an open access article distributed in accordance with the Creative Commons Attribution Non Commercial (CC BY-NC 4.0) license, which permits others to distribute, remix, adapt, build upon this work non-commercially, and license their derivative works on different terms, provided the original work is properly cited, appropriate credit is given, any changes made indicated, and the use is non-commercial. See: http://creativecommons.org/licenses/by-nc/4.0/.

ORCID iD

Mao Xiang Zhao http://orcid.org/0000-0001-7027-5913

\section{REFERENCES}

1 Liu L-S, Writing Group of 2010 Chinese Guidelines for the Management of Hypertension. [2010 Chinese guidelines for the management of hypertension]. Zhonghua Xin Xue Guan Bing Za Zhi 2011;39:579-615.

2 Chen W-W, Gao R-L, Liu L-S, et al. China cardiovascular diseases report 2015: a summary. J Geriatr Cardiol 2017;14:1-10.

3 Palatini P, Graniero GR, Mormino P, et al. Relation between physical training and ambulatory blood pressure in stage I hypertensive subjects. Results of the harvest trial. hypertension and ambulatory recording Venetia study. Circulation 1994;90:2870-6.

4 Borresen J, Lambert MI. Autonomic control of heart rate during and after exercise : measurements and implications for monitoring training status. Sports Med 2008;38:633-46.

5 Bellenger CR, Fuller JT, Thomson RL, et al. Monitoring athletic training status through autonomic heart rate regulation: a systematic review and meta-analysis. Sports Med 2016;46:1461-86.

6 Daanen HAM, Lamberts RP, Kallen VL, et al. A systematic review on heart-rate recovery to monitor changes in training status in athletes. Int J Sports Physiol Perform 2012;7:251-60.

7 Kovar D, Cannon CP, Bentley JH, et al. Does initial and delayed heart rate predict mortality in patients with acute coronary syndromes? Clin Cardiol 2004;27:80-6.

8 Diaz A, Bourassa MG, Guertin M-C, et al. Long-Term prognostic value of resting heart rate in patients with suspected or proven coronary artery disease. Eur Heart J 2005;26:967-74.

9 Mauss O, Klingenheben T, Ptaszynski P, et al. Bedside risk stratification after acute myocardial infarction: prospective evaluation of the use of heart rate and left ventricular function. $J$ Electrocardiol 2005;38:106-12.

10 Prasada S, Oswalt C, Yeboah P, et al. Heart rate is an independent predictor of all-cause mortality in individuals with type 2 diabetes: the diabetes heart study. World J Diabetes 2018:9:33-9.

11 Gillman MW, Kannel WB, Belanger A, et al. Influence of heart rate on mortality among persons with hypertension: the Framingham study. Am Heart J 1993;125:1148:1154

12 Benetos A, Rudnichi A, Thomas F, et al. Influence of heart rate on mortality in a French population: role of age, gender, and blood pressure. Hypertension 2012;33:44-52.

13 Palatini P, Thijs L, Staessen JA, et al. Predictive value of clinic and ambulatory heart rate for mortality in elderly subjects with systolic hypertension. Arch Intern Med 2002;162:2313.

14 Julius S, Palatini P, Kjeldsen SE, et al. Usefulness of heart rate to predict cardiac events in treated patients with high-risk systemic hypertension. Am J Cardiol 2012;109:685-92.

15 Paul L, Hastie CE, Li WS, et al. Resting heart rate pattern during follow-up and mortality in hypertensive patients. Hypertension 2010;55:567-74.

16 Thomas F, Bean K, Provost JC, et al. Combined effects of heart rate and pulse pressure on cardiovascular mortality according to age. $J$ Hypertens 2001;19:863-9.

17 Williams B, Mancia G, Spiering W, et al. 2018 ESC/ESH guidelines for the management of arterial hypertension: the task force for the management of arterial hypertension of the European Society of cardiology and the European Society of hypertension. J Hypertens 2018.

18 Zhongwei S, Yinqin W, Jinxiu L, et al. Chinese expert consensus on heart rate management for hypertensive patients[J]. Chinese Journal of The Frontiers of Medical Science(Electronic Version) 2017;9:29-36.

19 Zhao Q, Li H, Wang A, et al. Cumulative resting heart rate exposure and risk of all-cause mortality: results from the Kailuan cohort study. Sci Rep 2017;7:40212.

20 Li Z, Wang A, Cai J, et al. Impact of proteinuria and glomerular filtration rate on risk of ischaemic and intracerebral hemorrhagic stroke: a result from the Kailuan study. Eur J Neurol 2015;22:355-60.
21 Wang A, Wu J, Zhou Y, et al. Measures of adiposity and risk of stroke in China: a result from the Kailuan study. PLoS One 2013;8:e61665.

22 Peng M, Wu S, Jiang X, et al. Long-Term alcohol consumption is an independent risk factor of hypertension development in northern China: evidence from Kailuan study. $J$ Hypertens 2013;31:2342:2347.

23 Liu Y, Jin C, Xing A, et al. Serum uric acid levels and the risk of impaired fasting glucose: a prospective study in adults of North China. PLoS One 2013;8:e84712.

24 Wang A, Zhang Y, Li J, et al. High SBP trajectories are associated with risk of all-cause death in general Chinese population. $J$ Hypertens 2018;36:1299-305.

25 Wang A, Chen S, Wang C, et al. Resting heart rate and risk of cardiovascular diseases and all-cause death: the Kailuan study. PLoS One 2014:9:e110985.

26 Cubbon RM, Ruff N, Groves D, et al. Ambulatory heart rate range predicts mode-specific mortality and hospitalisation in chronic heart failure. Heart 2016;102:223-9.

27 Steinberg BA, Kim S, Thomas L, et al. Increased heart rate is associated with higher mortality in patients with atrial fibrillation (AF): results from the outcomes Registry for better informed treatment of AF (ORBIT-AF). J Am Heart Assoc 2015;4.

28 Writing group of 2018 Chinese guidelines for the management of hypertension. guidelines for the prevention and treatment of hypertension in China 2018. Prevention and Treatment of CardioCerebral-Vascular Disease 2019;19:1-45.

29 Umemura S, Arima H, Arima S, et al. The Japanese Society of hypertension guidelines for the management of hypertension (JSH 2019). Hypertens Res 2019;42:1235-481.

30 Whelton PK, Carey RM, Aronow WS, et al. 2017 ACC/AHA/AAPA/ ABC/ACPM/AGS/APhA/ASH/ASPC/NMA/PCNA guideline for the prevention, detection, evaluation, and management of high blood pressure in adults. J Am Coll Cardiol 2017;71:213-21.

31 National Institute for Health and Clinical Excellence (NICE). Hypertension in adults: diagnosis and management [M/OL], (2016-11). Available: http://www.nice.org.uk/guidance/CG127 [Accessed 26 Nov 2016].

32 Nerenberg KA, Zarnke KB, Leung AA, et al. Hypertension Canada's 2018 guidelines for diagnosis, risk assessment, prevention, and treatment of hypertension in adults and children. Can J Cardiol 2018;34:506-25.

33 Wiysonge CS, Bradley HA, Volmink J, et al. Beta-Blockers for hypertension. Cochrane Database Syst Rev 2017;1:CD002003.

34 Reboussin DM, Allen NB, Griswold ME, et al. Systematic review for the 2017 ACC/AHA/AAPA/ABC/ACPM/AGS/APhA/ASH/ASPC/ NMA/PCNA guideline for the prevention, detection, evaluation, and management of high blood pressure in adults: a report of the American College of Cardiology/American heart association Task force on clinical practice guidelines. Circulation. In Press 2017.

35 Jamerson KA, Julius S, Gudbrandsson T, et al. Reflex sympathetic activation induces acute insulin resistance in the human forearm. Hypertension 1993;21:618-23.

36 Palatini P, Mos L, Santonastaso M, et al. Resting heart rate as a predictor of body weight gain in the early stage of hypertension. Obesity 2011;19:618-23.

37 Reaven GM, Lithell H, Landsberg L. Hypertension and associated metabolic abnormalities--the role of insulin resistance and the sympathoadrenal system. N Engl J Med 1996;334:374-82.

38 Shimazu T. Innervation of the liver and glucoregulation: roles of the hypothalamus and autonomic nerves. Nutrition 1996;12:65-6.

39 Nonogaki K. New insights into sympathetic regulation of glucose and fat metabolism. Diabetologia 2000;43:533-49.

40 Caetano J, Delgado Alves J, Alves JD. Heart rate and cardiovascular protection. Eur J Intern Med 2015;26:217-22.

41 Whelton SP, Blankstein R, Al-Mallah $\mathrm{MH}$, et al. Association of resting heart rate with carotid and aortic arterial stiffness: multi-ethnic study of atherosclerosis. Hypertension 2013;62:477-84.

42 Wang L, Cui L, Wang Y, et al. Resting heart rate and the risk of developing impaired fasting glucose and diabetes: the Kailuan prospective study. Int J Epidemiol 2015;44:689-99.

43 Wang A, Liu X, Guo X, et al. Resting heart rate and risk of hypertension: results of the Kailuan cohort study. $J$ Hypertens 2014;32:1600-5.

44 Bangalore S, Messerli FH, FS O, et al. The association of admission heart rate and in-hospital cardiovascular events in patients with nonST-segment elevation acute coronary syndromes: results from 135 164 patients in the crusade quality improvement initiative. Faseb $J$ 2004;18:400-2. 